\title{
EARLY-TYPE GALAXIES IN THE COMA CLUSTER: A NEW PIECE IN THE CALCIUM PUZZLE
}

\author{
A. J. Cenarro, ${ }^{1}$ P. Sánchez-Blázquez, N. Cardiel, ${ }^{2}$ and J. Gorgas \\ Departamento de Astrofísica, Facultad de Físicas, Universidad Complutense de Madrid, 28040 Madrid, Spain; cen@astrax.fis.ucm.es \\ Received 2004 January 16; accepted 2004 September 10; published 2004 September 16
}

\begin{abstract}
We present measurements of the Ca II triplet and the Ca4227 Lick index for a sample of early-type galaxies in the Coma Cluster, deriving, for the first time, their corresponding relationships with velocity dispersion. Compared with a similar subsample of elliptical galaxies in the field, Coma galaxies with velocity dispersions in the range $\approx 180-270 \mathrm{~km} \mathrm{~s}^{-1}$ exhibit significant differences in the strengths of the Ca features, suggesting an influence of the environment on the star formation histories of these galaxies. We argue that the main scenarios previously proposed to explain the relatively low $\mathrm{Ca}$ II triplet of galaxies are not able by themselves to simultaneously reconcile the strengths of the two $\mathrm{Ca}$ indices in both environments.
\end{abstract}

Subject headings: galaxies: abundances — galaxies: clusters: general — galaxies: evolution — galaxies: formation - galaxies: stellar content

\section{INTRODUCTION}

The near-infrared (NIR) Ca II triplet constitutes the strongest absorption feature in the integrated spectra around $8600 \AA$ of relatively old stellar populations. Given its well-known capability to derive the metallicity of Galactic globular clusters (Armandroff \& Zinn 1988, hereafter A88; Rutledge et al. 1997), this spectral feature was considered a useful tool to study the stellar populations of normal galaxies. However, the first observational work (Cohen 1979; Bica \& Alloin 1987; Terlevich et al. 1990; Houdashelt 1995) reported only small differences among the integrated $\mathrm{Ca}$ II triplet of several types of galaxies, remarkably at variance with the trends found when using other metallicity indicators (e.g., the $\mathrm{Mg}$ triplet at $5175 \AA$ ). The difficulties of previous single stellar population (SSP) models to make reliable predictions of the Ca II triplet (due to severe deficiencies in the available stellar libraries) prevented an understanding of the above apparent inconsistency.

After a series of papers devoted to constructing realistic models for disentangling the actual behavior of the $\mathrm{Ca}$ II triplet in stellar populations (Cenarro et al. 2001a, hereafter C01; Cenarro et al. 2001b; Cenarro et al. 2002, hereafter C02; Vazdekis et al. 2003, hereafter V03), the above topic is being revisited and constitutes a controversial matter of debate. There exist recent evidences showing that, unlike other metal lines, the $\mathrm{Ca}$ II triplet is surprisingly anticorrelated with central velocity dispersion $\left(\sigma_{0}\right)$ for several types of galaxies: elliptical galaxies (E's) in the field (Saglia et al. 2002, hereafter S02; Cenarro et al. 2003, hereafter C03), bulges of spiral galaxies (FalcónBarroso et al. 2003, hereafter F03), and dwarf E's in the Fornax Cluster (Michielsen et al. 2003). Even more importantly, the measured values in giant E's lie well below model predictions for any reasonable choice of stellar population parameters (age and metallicity). A wide range of interpretations, like the existence of a dwarf-enriched stellar population, Ca underabundances, $\mathrm{Ca}$ depletion in the interstellar medium, or a composite stellar population have been discussed in the above references. Unfortunately, the lack of definitive evidences allows no consensus to exist at the moment.

\footnotetext{
'Also at UCO/Lick Observatory, University of California, Santa Cruz, CA 95064.

${ }^{2}$ Also at Calar Alto Observatory, CAHA, Apartado 511, 04044 Almeria, Spain.
}

In the blue spectral range, $\mathrm{Ca} 4227$ is considered the most Ca-sensitive Lick index (Tripicco \& Bell 1995). Intriguingly, despite $\mathrm{Ca}$ being an $\alpha$-element like $\mathrm{Mg}$, the metallicities inferred for E's using Ca4227 suggest that $\mathrm{Ca}$ is not enhancedit could be even depressed-with respect to Fe (Worthey 1992; Vazdekis et al. 1997; Worthey 1998; Trager et al. 1998; Vazdekis et al. 2001; Proctor \& Sansom 2002, hereafter P02). Once again, the existence of $\mathrm{Ca}$ underabundances has been demanded to explain the low Ca4227 values (Thomas et al. 2003b, hereafter T03b).

It is well known that the environment is expected to play a decisive role in the assembling and star formation history of galaxies. Hierarchical scenarios predict that E's in rich clusters were mostly formed at high redshifts, whereas field E's may have experienced an extended and more complex star formation history (Kauffmann \& Charlot 1998). Although no differences in the $[\mathrm{Mg} / \mathrm{Fe}]$ ratios seem to exist between cluster and field E's (Jørgensen 1999; Kuntschner et al. 2002), Sánchez-Blázquez et al. (2003, hereafter S03) have recently found significant differences in $\mathrm{C}$ and $\mathrm{N}$ abundance ratios between Coma and field $\mathrm{E}$ 's. These differences impose strong constraints to models of galaxy formation and chemical evolution.

The above picture motivated us to extend the previous work to the analysis of the $\mathrm{Ca}$ II triplet and the $\mathrm{Ca} 4227$ index in galaxies of different environments: first of all, because it might reveal new clues to the so confusing $\mathrm{Ca}$ II triplet behavior, and second, because the fact that $\mathrm{Ca}$ seems to be an anomalous $\alpha$ element makes it especially interesting from the point of view of chemical evolution.

\section{OBSERVATIONS AND DATA REDUCTION}

This work makes use of long-slit spectroscopic data for two samples of galaxies: E's from the Coma Cluster and E's in the field. Both were observed during two different runs (1999 March and 2001 April) using ISIS, the double-arm spectrograph at the $4.2 \mathrm{~m}$ William Herschel Telescope (Observatorio del Roque de los Muchachos, La Palma). All details about the sample, observations, and data reduction for E's in the field are given in C03 and Sánchez-Blázquez (2004, hereafter S04) for the NIR and blue-arm data, respectively. We also refer the reader to $\mathrm{S} 04$ for a description of the reduction of the blue spectroscopic data for the Coma galaxies. In this section, we just concentrate on their NIR spectral range (8355-9164 ̊). 
Our sample of galaxies from the Coma Cluster consists of 28 early-type galaxies (E to S0) spanning a wide range of central velocity dispersions $\left(80 \mathrm{~km} \mathrm{~s}^{-1} \lesssim \sigma_{0} \lesssim 370 \mathrm{~km} \mathrm{~s}^{-1}\right.$ ). We have rejected the faintest low-mass galaxies in S03 because of quite large errors. When it was feasible, the 3.5 long slit ( $2^{\prime \prime}$ width, providing $2.9 \AA$ FWHM spectral resolution) was rotated to include two galaxies in the same exposure. Otherwise, single galaxies were observed by aligning the slit with the major axis. Exposure times of 1200-2000 s per galaxy led to signal-to-noise ratios from 30 to $120 \AA^{-1}$ in a central aperture of radius $R_{\text {eff }} / 8$.

Standard spectroscopic reduction procedures were performed with REDUCEME (Cardiel 1999). Apart from an accurate correction for the fringe pattern, we took special care on the sky subtraction and the correction for telluric absorptions at $\lambda \gtrsim 8950 \AA$ (by using the telluric pattern derived from the spectra of flux standard stars). Both are especially critical for Coma galaxies since the redshift of the cluster $(z \sim 0.025)$ places some index bandpasses in regions affected by atmospheric absorptions and strong sky emission lines. The availability of error spectra for each galaxy frame allowed us to estimate reliable uncertainties in the measurements of the indices. Index errors account for photon statistics, uncertainties in the flux calibration, and radial velocity determinations (see details in C01).

Final spectra were relative-flux-calibrated using spectrophotometric standard stars observed several times at different air masses. In order to transform the NIR spectra to the V03 spectrophotometric system, a sample of 39 stars (from B to late M spectral types) in common with $\mathrm{C} 01$ were observed during twilights. Following C03, they were also employed as templates for $\sigma_{0}$ determinations. Rather than using template-dependent polynomials, systematic differences between indices measured at different spectral resolutions were prevented by broadening all the spectra up to the largest $\sigma_{0}$ of the galaxy sample, namely, $370 \mathrm{~km} \mathrm{~s}^{-1}$, corresponding to NGC 4889.

The observation of four E's in common with $\mathrm{C} 03$ allowed us to confirm a homogeneous spectrophotometric system for the blue and NIR spectra of both observing runs.

\section{INDEX- $\sigma_{0}$ RELATIONS}

We analyze, for the first time, the behaviors of the $\mathrm{Ca}$ II triplet, the $\mathrm{H}$ Paschen series $\left(\mathrm{CaT}^{*}\right.$, CaT, and PaT line-strength indices; see definitions in C01), and the Ca4227 Lick index as a function of $\sigma_{0}$ for E's in the Coma Cluster. All the measurements correspond to a central aperture of radius $R_{\text {eff }} / 8$ (or $1^{\prime \prime}$ for galaxies with $R_{\text {eff }}<8^{\prime \prime}$ ). There exists a database ${ }^{3}$ listing the galaxy sample as well as the indices and $\sigma_{0}$ determinations.

Figure 1 shows the measurements of the above indices versus $\sigma_{0}$ for E's in the Coma Cluster and, for comparison, for a similar subsample of E's in the field. Error-weighted, least-squares linear fits to all data have been computed for both samples (see labels in Fig. 1). CaT* and CaT in Coma E's exhibit an anticorrelation with $\sigma_{0}$ (Figs. $1 a$ and $1 b$ ), which is compatible with the one derived for field E's (C03). However, because of the errors of the computed slopes, we cannot rule out that a flat trend with $\sigma_{0}$ could exist for the Coma sample. As in the case of field E's, a flat behavior is derived for the PaT index (Fig. 1c), while both slightly positive and negative (nearly flat) trends are evidenced for the Ca4227 indices of field and Coma E's, respectively (Fig. 1d). In brief, as regards to the qualitative

${ }^{3}$ See http://www.ucm.es/info/Astrof/ellipt/CATRIPLET.html.

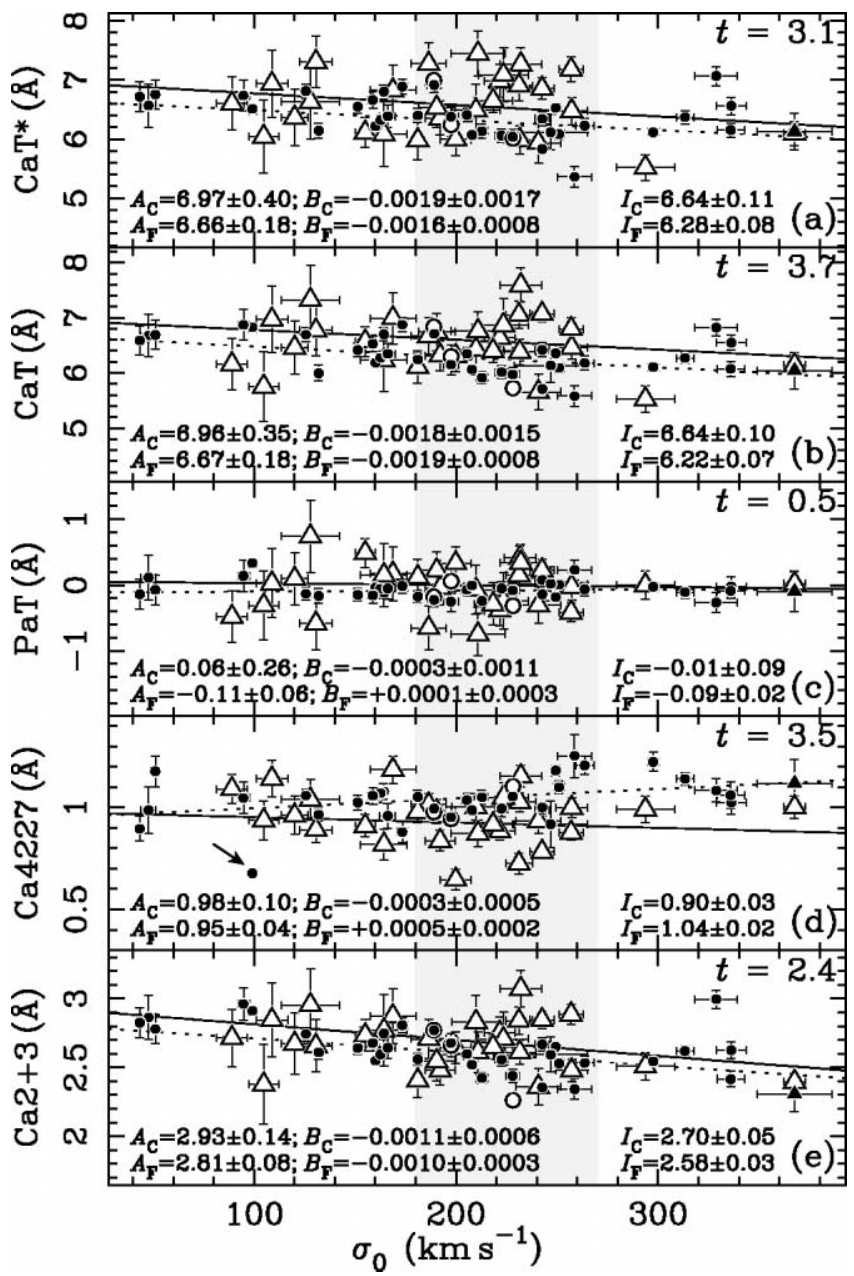

FIG. 1.-CaT*, CaT, PaT, Ca4227 and $\mathrm{Ca} 2+3$ ( $\equiv \mathrm{Ca} 2+\mathrm{Ca} 3$ indices by A88) vs. central velocity dispersion for E's in the Coma Cluster (triangles) and in the field (circles). For comparison, the indices of four galaxies in common between both runs are included (open circles and filled triangle). All the indices are measured at $370 \mathrm{~km} \mathrm{~s}^{-1}$ spectral resolution and corrected to the system defined by the models (V03). Solid (Coma) and dotted (field) lines represent errorweighted, least-squares linear fits to all data $\left(I=A+B \sigma_{0}\right.$; see the labels, with $\mathrm{C}$ and $\mathrm{F}$ subscripts referring to Coma and field E's). $I_{\mathrm{C}}$ and $I_{\mathrm{F}}$ are error-weighted mean indices for Coma and field E's within the range $180 \mathrm{~km} \mathrm{~s}^{-1} \lesssim \sigma_{0} \lesssim 270$ $\mathrm{km} \mathrm{s}^{-1}$ (shaded region). A $t$-value larger than $\sim 1.96$ indicates that $I_{\mathrm{C}}$ and $I_{\mathrm{F}}$ are statistically different. NGC 4742 (see arrow in panel $d$ ), with a very low Ca4227 because of a central young stellar population (see $\mathrm{C} 03$ ), has been rejected from the linear fits and the mean index computations.

behaviors with $\sigma_{0}$ of the above indices, we find no important differences between field and Coma E's.

The most striking differences between both samples arise from the mean absolute values of their $\mathrm{Ca}$ indices. In particular, although intermediate-low mass E's $\left(\sigma_{0} \lesssim 180 \mathrm{~km} \mathrm{~s}^{-1}\right)$ in both samples exhibit similar mean index values, we find clear differences for those E's within the range $180 \mathrm{~km} \mathrm{~s}^{-1} \lesssim \sigma_{0} \lesssim$ $270 \mathrm{~km} \mathrm{~s}^{-1}$ (in the following, we will refer to them as massive E's, the ones being the main subject of discussion in this Letter). Note that, other than visual inspection, no a priori argument exists to justify the above subsample selection. Interestingly, the fact that the differences in $\mathrm{C} 4668$ and $\mathrm{CN}_{2}$ for Coma and field E's are also particularly evident in a similar range of velocity dispersion (S03) supports the idea that massive E's in different environments could indeed have different stellar population properties. Unfortunately, the scarcity of E's with $\sigma_{0} \gtrsim 270 \mathrm{~km} \mathrm{~s}^{-1}$ in our Coma sample prevents us from confirming whether the above behavior extends to the high-mass end of the E family. 


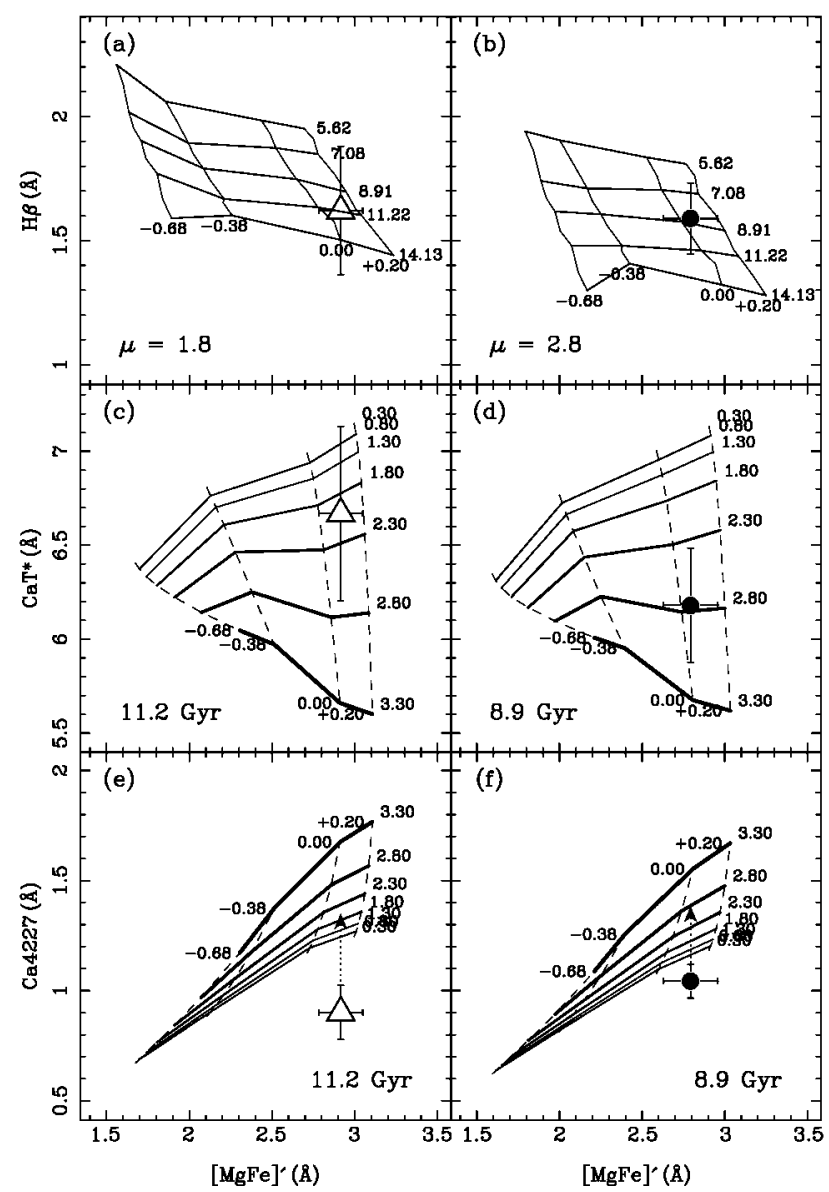

Fig. 2.-Mean values of $\mathrm{H} \beta$, [MgFe]', Ca4227, and $\mathrm{CaT}^{*}$ for Coma (open triangles) and field (filled circles) E's within the range $180 \mathrm{~km} \mathrm{~s}^{-1} \lesssim$ $\sigma_{0} \lesssim 270 \mathrm{~km} \mathrm{~s}^{-1}$. Rather than typical errors, error bars correspond to the rms standard deviation of the data (larger than the former ones). SSP model predictions by V03 and V99 at $370 \mathrm{~km} \mathrm{~s}^{-1}$ spectral resolution, respectively, are overplotted. In panels $a$ and $b$, age and metallicity vary from 5.62 to $14.13 \mathrm{Gyr}$ and from -0.68 to $+0.20 \mathrm{dex}$ at different Salpeter-like IMF slopes ( $\mu$; see the labels). Panels $c-f$ exhibit model predictions at fixed age (see the labels) with varying $\mu(0.3-3.3)$ and metallicity (as in panels $a$ and $b$ ). Arrows in panels $e$ and $f$ are explained in the text.

For this subsample of massive galaxies consisting of 17 Coma E's and 15 E's in the field, we have computed errorweighted means of the indices and their corresponding standard errors (see labels in Fig. 1). The statistical significance of the differences between the mean indices of both samples is studied by using a $t$-test (see Fig. 1; for a significance level of $\alpha=$ $0.05, t$-values larger than 1.96 indicate that a significant difference exists). Apart from the fact that no significant differences are found for the PaT index (as expected for relatively old stellar populations; see V03), we determine the existence of an intriguing behavior: while field E's exhibit Ca4227 values significantly larger than Coma E's, the contrary occurs for the $\mathrm{CaT}^{*}$ and $\mathrm{CaT}$ indices. In order to check that the above results are not driven by uncertainties in the correction for NIR telluric absorptions, we also measured the $\mathrm{Ca} 2$ and $\mathrm{Ca} 3$ classical indices by $\mathrm{A} 88(\mathrm{Ca} 2+3 \equiv \mathrm{Ca} 2+\mathrm{Ca} 3)$. The high sensitivities of these indices to velocity dispersion and to the presence of Paschen lines (see C01) are not important when, as is the case, they are measured on the spectra of old stellar populations at equal spectral resolution. In turn, the major improvement lies in the fact that their continuum sidebands are located in regions free from telluric absorptions for the redshift of the cluster. The data in Figure 1 (panel $e$ ) prove that the trends and differences inferred from these indices are compatible with the ones derived for $\mathrm{CaT}^{*}$ and $\mathrm{CaT}$.

\section{DISCUSSION}

As it has been recently reported by $\mathrm{S} 02, \mathrm{C} 03$, and F03, single and composite stellar population models with solar abundance ratios $([\alpha / \mathrm{Fe}]=0.0)$ and a standard initial mass function (IMF) cannot reproduce the low Ca II values of massive galaxies by solely assuming age and metallicity variations. Among others, two alternate scenarios have been proposed: (1) the existence of $\mathrm{Ca}$ underabundances and (2) the existence of a dwarfenriched stellar population. In the following paragraphs, we explore in turn whether the above scenarios are consistent enough to explain simultaneously the discrepancy with the model predictions and the differences between distinct environments.

1. Even though there exist no evolutionary synthesis models for the $\mathrm{Ca}$ II triplet in which nonsolar abundances ratios are considered, the $\mathrm{Ca}$ underabundant hypothesis has been proposed as a plausible scenario to explain the low $\mathrm{CaT}$ of massive galaxies (S02; F03). Making use of stellar population models with variable element abundance ratios for the Lick indices (Thomas et al. 2003a, hereafter T03a), T03b suggest the existence of a Ca depletion, with respect to the rest of $\alpha$-elements, that increases with galaxy mass. Under this scenario, and assuming that the integrated $\mathrm{Ca}$ II triplet indeed traces the $\mathrm{Ca}$ abundance, the relative differences in the CaT of massive Coma and field E's should be naturally explained as differences in the levels of $\mathrm{Ca}$ underabundances, field E's being "more Ca underabundant" than Coma galaxies. However, there exists a strong contradiction in the fact that while mean Ca II indices of massive Coma E's are larger than of those in the field, the contrary occurs for Ca4227. Furthermore, taking into account that $\mathrm{Ca} 4227$ is affected by $\mathrm{C}$ and $\mathrm{N}$ abundances in the sense that the stronger $\mathrm{CN}$ is, the lower Ca4227 is (Schiavon et al. 2002; P02; T03a), and given that field E's exhibit CN overabundances larger than those in Coma E's (S03), the relative differences in Ca4227 cannot be explained by just $\mathrm{CN}$ effects either. In fact, if we consider such an effect, the actual difference between the Ca4227 strengths of both samples should be even larger than that in Figure 1d. In summary, just the existence of $\mathrm{Ca}$ underabundances and/or $\mathrm{CN}$ effects is unable to reconcile $\mathrm{Ca} 4227$ and $\mathrm{CaT}^{*}$ at the same time.

2. Since the Ca II triplet of giant stars is stronger than that of dwarf stars, the existence of a dwarf-enriched stellar population could account for the low values of massive E's. This scenario can be parametrized by means of a variation of the IMF slope ( $\mu$; assuming a Salpeter-like IMF). The evolutionary synthesis models by V03 predict that, for old SSPs with intermediate-to-high metallicities, $\mathrm{CaT}^{*}$ and $\mathrm{CaT}$ mainly depend on $\mu$ (they decrease with the increasing $\mu$ because of a larger dwarf-to-giant stars ratio). In the light of the above predictions, massive E's are interpreted to have both metallicities and dwarf-to-giant ratios larger than low-mass E's (C03).

In Figure 2, we make use of the models by Vazdekis (1999, hereafter V99) and V03 to compare Coma and field E's under this scenario. The only way to reconcile $\mathrm{H} \beta,[\mathrm{MgFe}]^{\prime}$ (the one being independent of typical $\alpha / \mathrm{Fe}$ ratios as defined by T03a), and $\mathrm{CaT}^{*}$ at the same time is by invoking different ages, metallicities, and dwarf-to-giant ratios. In turn, the latter also affects the derived ages and metallicities because of the nonnegligible dependence of $\mathrm{H} \beta$ to the dwarf-to-giant ratio. From 
Figures $2 a-2 d$, we derive similar metallicities (above solar) for both subsamples, a mean age for field E's lower ( 8.9 Gyr) than that for Coma E's ( 11.2 Gyr), and mean dwarf-to-giant ratios larger for the former $(\mu \simeq 2.8)$ than for the latter $(\mu \simeq$ 1.8). However, when this scenario is simultaneously applied to $\mathrm{Ca} 4227$, the model predictions fail to reproduce the locus of the galaxies (Fig. 2c). Therefore, even though relative differences between the $\mathrm{Ca}$ indices of Coma and field E's could be compatible with just variations of the dwarf-to-giant ratio (the larger $\mu$ is, the lower $\mathrm{CaT}^{*}$ is, and the larger Ca4227 is; Figs. $2 c-2 f$ ), this argument is not enough to reconcile absolute values of $\mathrm{CaT}^{*}$ and $\mathrm{Ca} 4227$ by itself.

At this point, one notices that the current status is not quite optimistic. Could it be that both scenarios coexist, driving an apparent inconsistency? Even though the sensitivity of Ca4227 to unusual dwarf-to-giant ratios is not negligible, age and metal abundance effects are mainly expected to govern its behavior. The arrows in Figures $2 e$ and $2 f$ indicate the offsets in Ca4227arising not only from the existence of $\mathrm{Ca}$ subabundances but also from possible dilution effects due to $\mathrm{CN}$ enhancementsrequired to reproduce the SSP properties in the previous panels. On the contrary, several arguments support the idea that the $\mathrm{Ca}$ II triplet is rather insensitive to $\mathrm{Ca}$ abundances. First, the $\mathrm{CaT}$ index does not correlate with stellar $[\mathrm{Ca} / \mathrm{Fe}]$ abundance ratios (C02). Also, for SSPs older than $\sim 3$ Gyr with intermediate-tohigh metallicities, the integrated $\mathrm{Ca}$ II triplet saturates (or even decreases for dwarf-enriched SSPs) with increasing metallicity (V03). This is indeed a by-product of the increasing luminosity weight of late $\mathrm{M}$ stellar types (with very weak $\mathrm{Ca}$ II lines and strong $\mathrm{TiO}$ bands in their spectra) as the temperature of the isochrone becomes colder. Therefore, just because of temperature effects, very metal rich stellar populations with high $\mathrm{Ca}$ abundances are expected to exhibit $\mathrm{CaT}$ values lower than if, for example, they were solar-abundant (see more details in V03). In this sense, Ca II triplet indices are expected to be sensitive to global metallicity rather than to $\mathrm{Ca}$ abundances.

To conclude, we report, for the first time, systematic differences between the integrated Ca II triplet and Ca4227 of E's in the Coma Cluster and in the field, which are difficult to reconcile at the same time. Different abundance ratios and/or dwarf-enriched stellar populations could be conspiring to produce apparently inconsistent $\mathrm{Ca}$ indices. As proposed in S03, these kind of differences are probably driven by the effect of the environment on chemical evolution and galaxy formation. Unfortunately, we are far from determining any definitive proof for distinct star formation histories in massive E's located at different environments; the fact is that the current picture of calcium in galaxies seems to go beyond the capabilities of the available SSP models. An extensive effort in the field of nucleosynthesis and stellar atmospheres is in demand to help us understand how different abundance ratios affect not only linestrength indices but also the physics and temperature of isochrones. In the meantime, the collection of more and highquality data of $\mathrm{Ca}$ line strengths is crucial for trying to understand the controversial $\mathrm{Ca}$ puzzle.

The authors are indebted to the referee, S. M. Faber, for useful comments and suggestions. A. J. C. acknowledges financial support from a Universidad Complutense de Madrid Fundación del Amo Fellowship. The William Herschel Telescope is operated on the island of La Palma by the Royal Greenwich Observatory at the Observatorio del Roque de los Muchachos of the Instituto de Astrofísica de Canarias. This work was supported by the Spanish research project AYA200301840 .

\section{REFERENCES}

Armandroff, T. E., \& Zinn, R. 1988, AJ, 96, 92 (A88)

Bica, E., \& Alloin, D. 1987, A\&A, 186, 49

Cardiel, N. 1999, Ph.D. thesis, Univ. Complutense, Madrid

Cenarro, A. J., Cardiel, N., Gorgas, J., Peletier, R. F., Vazdekis, A., \& Prada, F. 2001a, MNRAS, 326, 959 (C01)

Cenarro, A. J., Gorgas, J., Cardiel, N., Pedraz, S., Peletier, R. F., \& Vazdekis, A. 2001b, MNRAS, 326, 981

Cenarro, A. J., Gorgas, J., Cardiel, N., Vazdekis, A., \& Peletier, R. F. 2002, MNRAS, 329, 863 (C02)

Cenarro, A. J., Gorgas, J., Vazdekis, A., Cardiel, N., \& Peletier, R. F. 2003, MNRAS, 339, L12 (C03)

Cohen, J. G. 1979, ApJ, 228, 405

Falcón-Barroso, J., Peletier, R. F., Vazdekis, A., \& Balcells, M. 2003, ApJ, 588, L17 (F03)

Houdashelt, M. L. 1995, Ph.D. thesis, Ohio State Univ.

Jørgensen, I. 1999, MNRAS, 306, 607

Kauffmann, G., \& Charlot, S. 1998, MNRAS, 294, 705

Kuntschner, H., Smith, R., Colles, M., Davies, R., Kaldare, R., \& Vazdekis, A. 2002, MNRAS, 337, 172

Michielsen, D., De Rijcke, S., Dejonghe, H., Zeilinger, W. W., \& Hau, G. K. T. 2003, ApJ, 597, L21

Proctor, R. N., \& Sansom, A. E. 2002, MNRAS, 333, 517 (P02)

Rutledge, G. A., Hesser, J. E., \& Stetson, P. B. 1997, PASP, 109, 907
Saglia, R. P., Maraston, C., Thomas, D., \& Bender, R. 2002, ApJ, 579, L13 (SO2)

Sánchez-Blázquez, P. 2004, Ph.D. thesis, Univ. Complutense, Madrid (S04)

Sánchez-Blázquez, P., Gorgas, J., Cardiel, N., Cenarro, J., \& González, J. J. 2003, ApJ, 590, L91 (S03)

Schiavon, R. P., Faber, S. M., Castilho, B. V., \& Rose, J. A. 2002, ApJ, 580, 850

Terlevich, E., Díaz, A. I., \& Terlevich, R. 1990, MNRAS, 242, 271

Thomas, D., Maraston, C., \& Bender, R. 2003a, MNRAS, 339, 897 (T03a) 2003b, MNRAS, 343, 279 (T03b)

Trager, S. C., Worthey, G., Faber, S. M., Burstein, D., \& González, J. J. 1998, ApJS, 116, 1

Tripicco, M. J., \& Bell, R. A. 1995, AJ, 110, 3035

Vazdekis, A. 1999, ApJ, 513, 224 (V99)

Vazdekis, A., Cenarro, A. J., Gorgas, J., Cardiel, N., \& Peletier, R. F. 2003, MNRAS, 340, 1317 (V03)

Vazdekis, A., Kuntschner, H., Davies, R., Arimoto, N., Nakamura, O., \& Peletier, R. 2001, ApJ, 551, L127

Vazdekis, A., Peletier, R. F., Beckman, J. E., \& Casuso, E. 1997, ApJS, 111, 203

Worthey, G. 1992, Ph.D. thesis, Univ. Calif., Santa Cruz

- 1998, PASP, 110, 888 